\title{
Clinical Experience and Dosimetry Outcome in Treating Breast Cancer with Field-in-Field Technique
}

\author{
${\text { Bidyapati Jha1,2, Hari Prasad Lamichhane², GisupNikha Prasiko3 }{ }^{3} \text {, Raju Prasad Srivastava4* }}^{*}$ \\ ${ }^{1}$ Department of Radiation Oncology, Bhaktapur Cancer Hospital, Dudhpati, Bhaktapur, Nepal \\ ${ }^{2}$ Central Department of Physics, Institute of Science \& Technology, Tribhuvan University, Kirtipur, Kathmandu, Nepal \\ ${ }^{3}$ Nepal Cancer Hospital and Research Center, Harsiddhi, Latitpur, Nepal \\ ${ }^{4}$ Department of Radiation Oncology, Ghent University Hospital, Corneel Heymanslaan, Ghent, Belgium \\ Email:bjha2000us@yahoo.com, ^rajupsrivasstava@gmail.com
}

How to cite this paper: Jha, B., Lamichhane, H.P., Prasiko, G. and Srivastava, R.P. (2021) Clinical Experience and Dosimetry Outcome in Treating Breast Cancer with Field-in-Field Technique. Advances in Breast Cancer Research, 10, 35-43.

https://doi.org/10.4236/abcr.2021.103003

Received: April 9, 2021

Accepted: June 6, 2021

Published: June 9, 2021

Copyright $\odot 2021$ by author(s) and Scientific Research Publishing Inc. This work is licensed under the Creative Commons Attribution International License (CC BY 4.0).

http://creativecommons.org/licenses/by/4.0/

\begin{abstract}
Purpose: The study is aimed to establish the dosimetric characteristics of field-in-field (FiF) technique for carcinoma of breast treatment in Nepal. We assumed that FIF technique may result in improved dose distribution and reduced acute toxicity in these patients. Methods: Forty breast cancer patients participated in this study. A total dose of $50 \mathrm{~Gy}$ in 25 fractions was prescribed to the planning target volume. FiF plan was generated in treatment planning system. Dose volume histograms were evaluated for PTV and organs at risks. Several parameters were analyzed for the PTVs and organ at risks (OARs) together with the Conformity index (CI), and the Homogeneity index (HI). Results: The dose coverage of breast volume was achieved. The $\mathrm{V}_{95 \%}$ (volume of $95 \%$ ) of PTV covered $\mathrm{D}_{95 \%}$ (Dose of $95 \%$ ). The PTV dose was covered to $49.98 \pm 0.9$ Gy and $49.81 \pm 1.1$ Gy for the left and right breast, respectively. The mean lung dose was $14.87 \pm 0.91 \mathrm{~Gy}$. The homogeneity index $(0.26 \pm 0.17$ and $0.22 \pm 0.13)$ and conformity index $(1.59 \pm 0.75$ and $1.36 \pm$ $0.45)$ were analyzed for left and right breast, respectively. Conclusion: The study supports the viability of FiF technique in the treatment of breast cancer in Nepal. The FIF technique enables better dose distribution in the PTV and reduces dose to OARs. The FiF technique provides dosimetric advantages and requires less planning time.
\end{abstract}

\section{Keywords}

Field in Field (FiF), Planning Target Volume (PTV), Treatment Planning System (TPS), Homogeneity Index (HI), Conformity Index (CI) 


\section{Introduction}

Breast cancer represents the most common cancer in women worldwide [1], constituting $24.2 \%$ of all malignancies, according to GLOBOCAN statistics [2]. The management of breast cancer is based on tumor and patient characteristics. It involves a multimodality approach and includes a combination of surgery, with or without radiation therapy or systemic therapy. The most common type of radiation used to treat breast cancer is external beam radiation therapy. Radiotherapy has an essential role in the management of breast cancer. The aim is to deliver homogeneous maximum dose to the planning target volume and a minimum dose to surrounding normal tissue.

In the last few decades, the treatment plan has been simply provided by a two-dimensional (2-D) image. With 2-D treatment, it is difficult to reach dose homogeneity because of the concave shape of the breast. The progress and developments in medical imaging, treatment planning system (TPS), and dosimetric devices have allowed achieving homogeneous dose distribution to PTV. In developing countries such as Nepal, three-dimensional conformal radiotherapy and Field-in-Field (FiF) are common radiotherapy technique to treat breast cancer.

Two opposite tangential beams are used in $3 \mathrm{D}$ radiation treatment in most cases. Physical, dynamic, and virtual wedges and compensators are commonly used in the conformal technique. The FIF technique uses several less-weighted fields with a small field size to optimize dose distribution. Several studies [3] [4], [5] have revealed that the FIF technique leads to a more satisfactory dose distribution in post-surgical radiotherapy of breast cancer, as compared to the conformal technique. An ideal plan is one in which the total planning target volume (PTV) is between the dose level of $95 \%$ and $107 \%$ dose levels relative to the $100 \%$ prescription dose (ICRU 50). The main trouble with tangential field breast irradiation lies in the achievement of homogeneous dose distribution inside the planning target volume.

The CT scanning and the accessibility of a three-dimensional treatment planning system have improved the delivery of radiation to the breast and peripheral lymphatic system efficiently [6]. With the convenience of a CT-scanner, most radiothreapy centers are gradually shifting toward a CT-based treatment planning system. CT-based 3D treatment planning allows the physicist and physician to evaluate the dosimetry across the PTV. Therefore, the plan could be optimized to limit the lung dose and other critical organ volumes with selective blocking. The hot spots could be minimized by using a higher energy and a smaller wedge angle.

This study focused on left and right breast treatment performed in order to evaluate the dose distribution using the FiF radiotherapy technique and correlate the normal tissue toxicity.

\section{Material and Methods}

\section{Patient selection:}

The study was carried out at the Radiation Oncology Department, Bhaktapur 
Cancer Hospital (BCH) Bhaktapur from August 2020 to January 2021 on 20 left side and 20 right side breast cancer patients. The patients eligible for the study and sociodemographic are described in Table 1 . The patients were categorized into three groups according to the thickness of their chest wall, as follows, small $(\leq 500 \mathrm{cc})$, medium $(500-800 \mathrm{cc})$ and large $(\geq 800 \mathrm{cc})$. All the patients were selected between 30 - 60 years old. Re-irradiation and pregnant patients have not been involved in the study.

\section{Simulator:}

All patients were scanned by computed tomography (CT) simulation (Siemens Somatom Definition Flash, Germany). The patient was positioned on the breast board in supine position with both hands raised above the head. The CT image data was acquired from the mandible to the $4^{\text {th }}$ lumbar vertebra, with slice thickness of $3 \mathrm{~mm}$ during normal breathing. The CT images were then transferred to the Eclipse (v15.6, Varian Medical Systems, Palo Alto, CA) Treatment Planning System (TPS).

\section{Linear accelerator:}

We used to treat our patients with a Cobalt-60 machine before installation of linear accelerator. A Varian linear accelerator (Varian Medical Systems, Palo Alto, CA) with TPS installed in 2019. The Clinic iX linear accelerator equipped with a 120 Millennium MLC, was used for treatment. The width of MLC was 0.5 $\mathrm{mm}$ at the isocenter.

\section{Treatment planning:}

The clinical tumor volume (CTV), PTV, and organs at risk such as contralateral breast, lungs, and heart on CT images were contoured by the radiation oncologist. The dose limits the organs at risk was defined according to our clinical protocol. The prescribed dose was $50.0 \mathrm{~Gy}$ in 25 fractions for all patients. Two opposing tangential beams were created, with the use of beam's-eye-view projections. The gantry angles were adjusted and shielding OAR was applied with the use of a multileaf collimator (MLC). The $2 \mathrm{~cm}$ leaf margin was added to the skin side. Photon beams of either 6 or $15 \mathrm{MV}$ were selected depending on the separation of fields. The main field was reproduced as the subfield and the multileaf

Table 1. Summary of sociodemographic and patient' data for breast treatment by FiF technique in Radiation Oncology Department, BCH Bhaktapur.

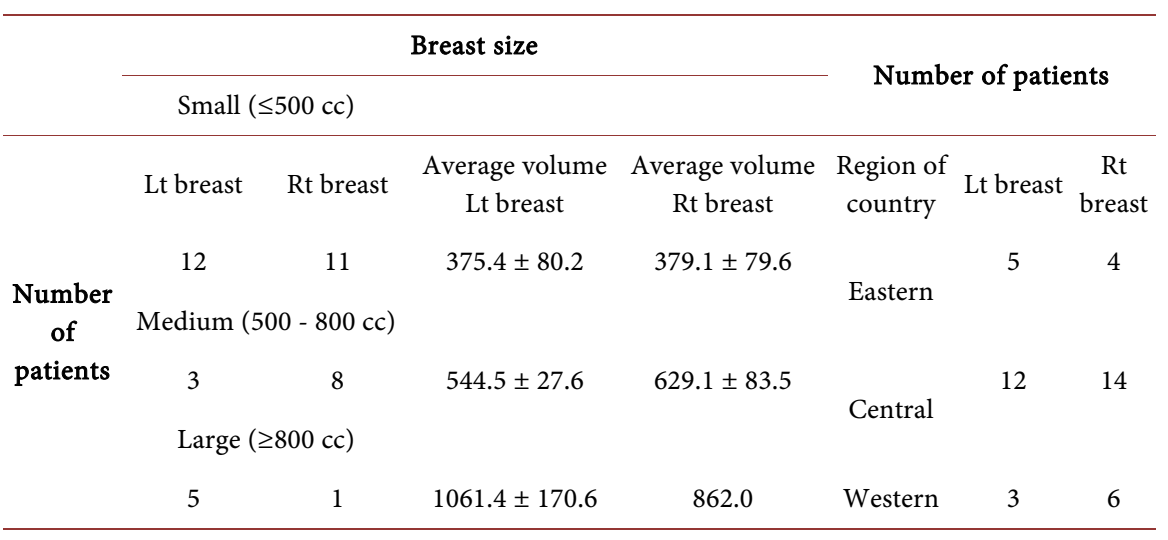


collimators (MLCs) were manipulated to shield the region of the breast receiving the hot spot. The beam weight of the subfield was $3 \%$ of the main field. The minimum monitor unit (MU) of each subfield was 8 . The FiF plan was generated in Eclipse TPS. The beams were used in the most optimal way for each patient to produce the best coverage of the PTV together with the minimum dose to the organs at risk.

\section{Dosimetric evaluation:}

A DVH was created for the CTV, PTV and the organs at risk (OARs) for each patient. The $\mathrm{D}_{98 \%}, \mathrm{D}_{95 \%}, \mathrm{D}_{2 \%}$ and $\mathrm{D}_{\max }$ were analyzed for PTV. The $\mathrm{D}_{95 \%}$ is the minimum dose received by $95 \%$ of PTV. The Treatment plan was evaluated by analyzing the conformity index (CI) and the homogeneity index (HI) as defined according to ICRU 83 report. $\mathrm{HI}$ is the fraction of the dose difference of $2 \%$ and $98 \%$ to the PTV to the dose of $50 \%\left(\mathrm{HI}=\left(\mathrm{D}_{2 \%}-\mathrm{D}_{98 \%}\right) / \mathrm{D}_{50 \%}\right)$. The value of $\mathrm{HI}$ zero indicates that the dose distribution is almost homogeneous. $\mathrm{CI}$ is the ration of treated volume (TV) to PTV (CI $\left.=\mathrm{TV}_{\text {prescribed }} / \mathrm{PTV}_{\text {volume }}\right)$. The parameters V20 and mean dose were analyzed for lung and $\mathrm{D}_{2 \%}$ (Dose to $2 \%$ volume) for heart dose.

Patient specific treatment was performed on a Varian EPID (Electronic Portal Imaging Devices). The gamma parameters were evaluated as a difference between the calculated and the measured dose distributions [7]. SPSS 21 was used for data analysis. The FiF plan parameters were tested for statistical significance using the t-test. The p-values $<0.05$ were considered as significant.

\section{Results}

The PTV volume of left and right breast treatments has been shown in Figure 1. The average PTVs were $572.4 \pm 326.6 \mathrm{cc}$ and $503.2 \pm 167.5 \mathrm{cc}$ for left and right breast respectively. The majority of the patients underwent mastectomy.

Figure 2 showed the left breast treatment planning in transverse, frontal and

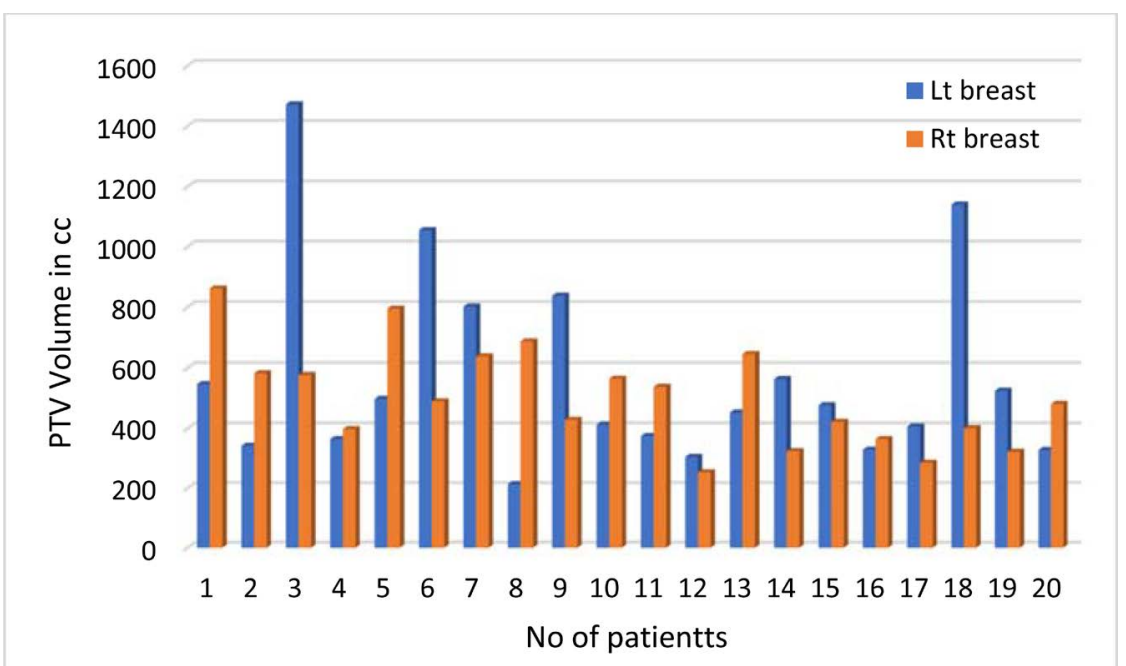

Figure 1. Shows the PTV volume of left and right breast treatments. The PTV volume was calculated by TPS in three dimensions. 


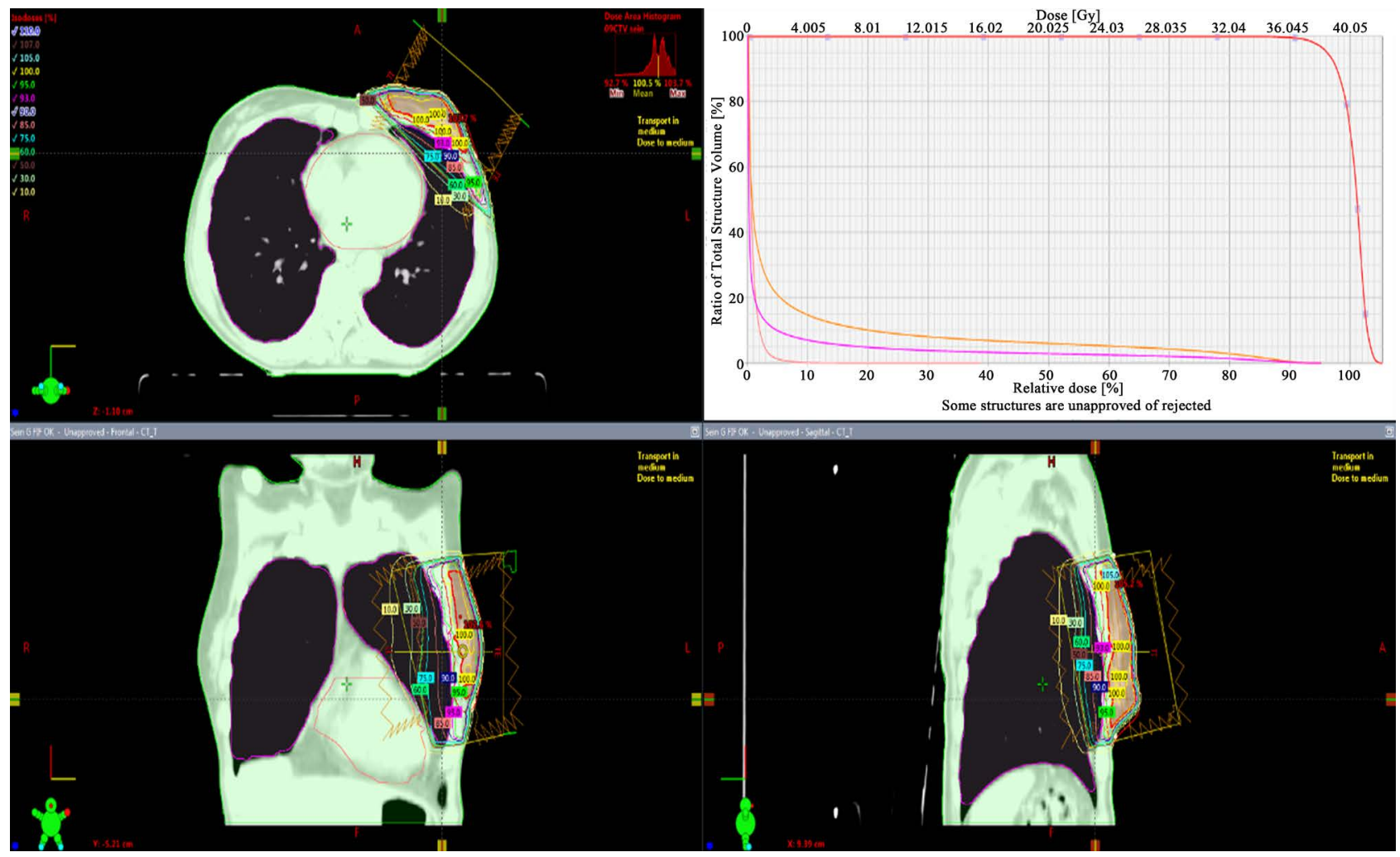

Figure 2. Shows the dose distribution for the left breast in transverse, frontal and sagittal views and dose volume histogram (DVH) presentation. Heart, Lungs are delineated as organ at risks.

sagittal views and DVH presentation. We were able to achieve good dose coverage. The $\mathrm{V}_{95 \%}$ (volume of $95 \%$ ) of PTV covered $\mathrm{D}_{95 \%}$ (Dose of $95 \%$ ). Our results revealed that the mean PTV dose was covered to $49.98 \pm 0.9$ Gy and $49.81 \pm 1.1$ Gy for the left and right breast respectively.

Table 2 summarizes the dosimetry outcome of PTV and OAR dose. The homogeneity and the conformity index were better in the right breast compared to the left breast because of breast volume. The $\mathrm{D}_{2 \%}$ of the heart was $4.5 \pm 0.89 \mathrm{~Gy}$ for the left breast. The mean dose of total lung was $14.23 \pm 3.4 \mathrm{~Gy}$. The OAR dose was followed according as the protocol.

According to QUANTEC, 2010 guidelines, V25 (volume of the target subjected to 25 Gy) should be less than $10 \%$ and V30 should be less than $46 \%$ to avoid cardiac complications. In the entire group of patients treated by the 3D-CRT technique, the V25 and V30 were both $<5 \%$ of the volume whether they had mastectomy or lumpectomy.

\section{Discussion}

Breast cancer treatment planning has progressed from the conventional tangential technique which was performed with the Cobalt-60 machine with the half-blocked breast cone to avoid divergence of the beam to the lung. Asymmetric jaws were used in the linear accelerator to avoid beam divergence to the lung. In developing countries like Nepal, patients come to hospital for treatments in 
Table 2. Dose volume constraints for PTV and organs at risk. Standard deviation is indicated as a statistical error uncertainty.

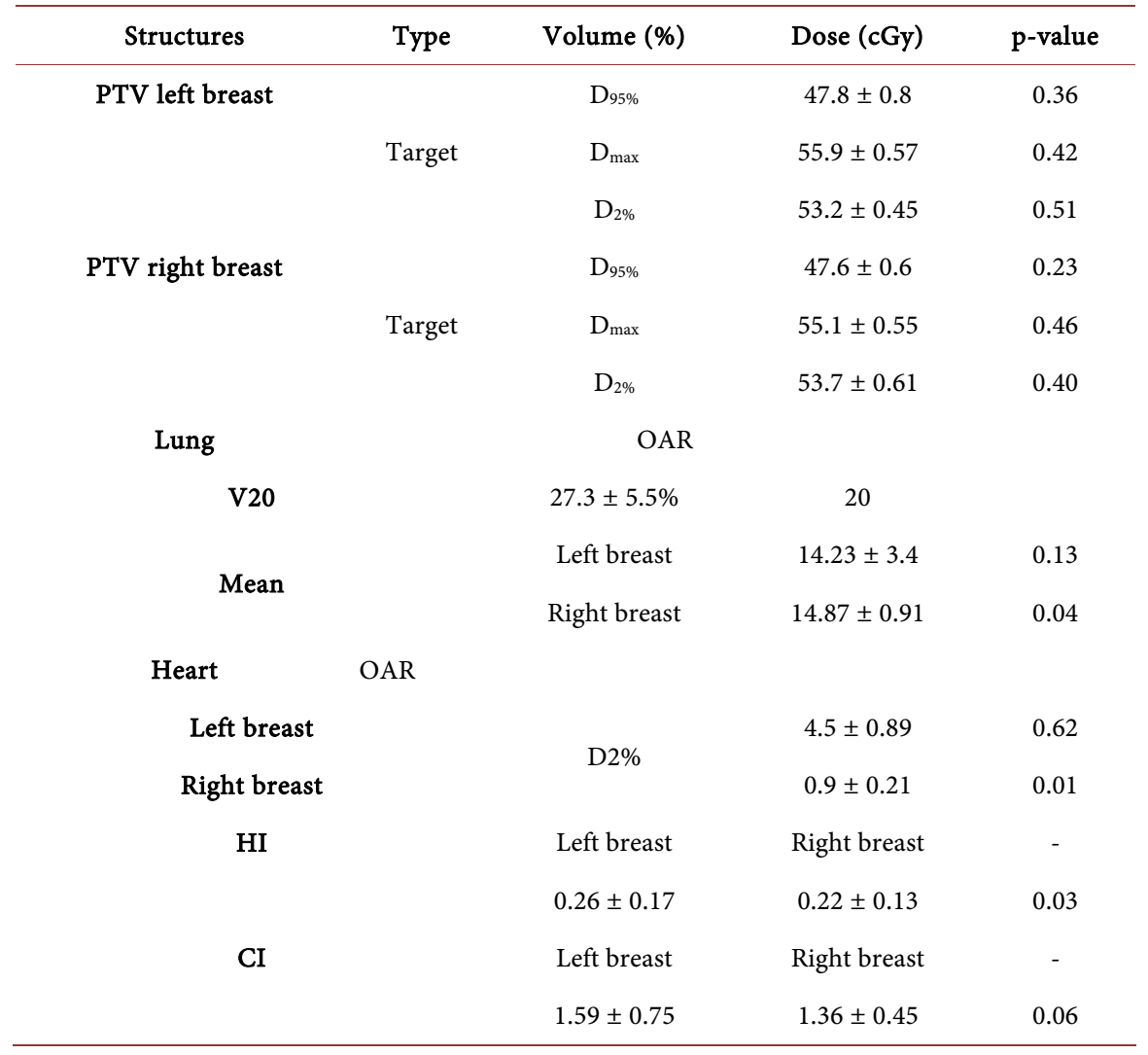

advanced stages of disease. It is not possible to have breast conserving surgery in the majority of patients. Therefore, mastectomy is mostly the choice of treatment. The geometric difference between the target volume of the chest wall and the whole breast is large and it has impact on the resulting dose distribution.

Smith et al. [8] studied tangential IMRT plans with 2D plans for the adjuvant radiotherapy of the whole breast with early breast cancer. They reported that the IMRT plans showed a significant improvement of the PTV, HI, heart and whole lung, compared to the conventional technique. Baycan et al. [9] compared FIF and 3D conformal of the left breast patients. They found significantly lower monitor unit $(\mathrm{MU})$, pulmonary and aorta dose. Our study revealed the similar outcomes.

Moorthy et al. [10] investigated the dosimetric features of IMRT in comparison with 3D-CRT for chest wall. There was an improvement in the conformity and homogeneity index.

Freedman et al. [11] stated that IMRT drops acute skin toxicity for women receiving radiation for breast cancer.

Borges C. et al. [12] examined comparatively different breast planning techniques and algorithms for radiation therapy treatment. Their results indicated a larger volume of OAR receiving a low dose when beams were set other than according to the tangential technique. Yavas [3] confirmed that the FIF technique 
lowered the maximum dose of the PTV, while delivering better dose distribution.

Hidekezu Tanaka [13] reported that the alternative subfield method (ASM) allows better dose distribution than a single pair of subfield method and to multiple pairs of subfield method (MSM) regardless of the breast size. Sasaoka and Futami [14] performed the dosimetric evaluation of whole breast radiotherapy using the FiF technique. They declared that the FiF technique reduced hot spots in PTV. We obtained similar results in our study.

Emami [15] studied the tolerance of normal tissue to therapeutic radiation and found out that symptomatic radiation pneumonia is one of the most common toxicities of breast cancer patients treated by radiotherapy. Darby SC [16] analyzed the risk of ischemic heart disease in women after radiotherapy for breast cancer. They concluded that the overall average of the mean doses to the whole heart was $4.9 \mathrm{~Gy}$ (for the range, from 0.03 to 27.72 ). There was a linear $7.4 \%$ per gray increase in major coronary events with no threshold. In our study, the mean dose was less than 5 Gy. Many studies [17] [18] have verified that IMRT plans scored better as compared to VMAT plans regarding mean the heart dose, low-dose parameter V5Gy, high-dose parameter V25Gy, and LAD dose.

Taylor C. [19], Hall E.J. 2006 [20] studied the risk of radiation induced second cancers. They concluded that the risk of radiation-induced secondary malignancies depends on the number of MUs, radiation scattered from the treatment volume etc. In our present study, MU is smaller as compared to that of IMRT plan. The combination of different techniques (i.e. hybrid) has been shown to exhibit advantages over conventional, IMRT, FIF, VMAT in minimizing the cardiac and the ipsilateral lung doses [21]. Our future research will focus on the hybrid technique for treatment of breast cancer.

\section{Conclusion}

The present study confirmed that the use of the FIF radiotherapy technique for breast treatment leads to homogeneous dose distribution in the planning target volume. The FiF technique provides dosimetric advantages and requires less planning time. Additionally, the implementation of this technique in the radiotherapy department is straight forward resulting in an overall dropping dose for OAR. However, our study has some limitations including small sample size and the lack of assessment of clinical parameters especially of OAR complications.

\section{Conflicts of Interest}

The authors declare no conflicts of interest regarding the publication of this paper.

\section{References}

[1] Forouzanfar, M.H., Foreman, K.J., Delossantos, A.M., Lozano, R., Lopez, A.D., Murray, C.J.L., et al. (2011) Breast and Cervical Cancer in 187 Countries between 1980 and 2010: A Systematic Analysis. The Lancet, 378, 1461-1484. 
https://doi.org/10.1016/S0140-6736(11)61351-2

[2] Bray, F., Ferlay, J., Soerjomataram, I., Siegel, R.L., Torre, L.A. and Jemal, A. (2018) Global Cancer Statistics 2018: GLOBOCAN Estimates of Incidence and Mortality Worldwide for 36 Cancers in 185 Countries. CA: A Cancer Journal for Clinicians, 68, 394-424. https://doi.org/10.3322/caac.21492

[3] Yavas, G., Yavas, C. and Acar, H. (2012) Dosimetric Comparison of Whole Breast Radiotherapy Using Field in Field and Conformal Radiotherapy Techniques in Early Stage Breast Cancer. International Journal of Radiation Research, 10, 131-138.

[4] Lee, J.-W., Hong, S., Choi, K.-S., Kim, Y.-L., Park, B.-M., Chung, J.-B., et al. (2008) Performance Evaluation of Field-in-Field Technique for Tangential Breast Irradiation. Japanese Journal of Clinical Oncology, 38, 158-163.

https://doi.org/10.1093/jjco/hym167

[5] Barnett, G.C., Wilkinson, J.S., Moody, A.M., Wilson, C.B., Twyman, N., Wishart, G.C., et al. (2012) Randomized Controlled Trial of Forward-Planned Intensity Modulated Radiotherapy for Early Breast Cancer: Interim Results at 2 Years. International Journal of Radiation Oncology, Biology, Physics, 82, 715-723.

https://doi.org/10.1016/j.ijrobp.2010.10.068

[6] Hartsell, W.F., Murthy, A.K., Kiel, K.D., Kao, M. and Hendrickson, F.R. (1990) Technique for Breast Irradiation Using Custom Blocks Conforming to the Chest Wall Contour. International Journal of Radiation Oncology, Biology, Physics, 19, 189-195. https://doi.org/10.1016/0360-3016(90)90153-B

[7] Depuydt, T., Van Esch, A. and Huyskens, D.P. (2002) A Quantitative Evaluation of IMRT Dose Distributions: Refinement and Clinical Assessment of the Gamma Evaluation. Radiotherapy and Oncology, 62, 309-319.

https://doi.org/10.1016/S0167-8140(01)00497-2

[8] Smith, W., Menon, G., Wolfe, N., Ploquin, N., Trotter, T. and Pudney, D. (2010) IMRT for the Breast: A Comparison of Tangential Planning Techniques. Physics in Medicine \& Biology, 55, 1231-1241. https://doi.org/10.1088/0031-9155/55/4/022

[9] Baycan, D., Karacetin, D., Balkanay, A.Y. and Barut, Y. (2012) Field-in-field IMRT versus 3D-CRT of the Breast. Cardiac Vessels, Ipsilateral Lung, and Contralateral Breast Absorbed Doses in Patients with Left-Sided Lumpectomy: A Dosimetric Comparison. Japanese Journal of Radiology, 30, 819-823.

https://doi.org/10.1007/s11604-012-0126-Z

[10] Moorthy, S., DasMajumdar, S.K., Elhateer, H., Mohan, R. and Mohammed, S. (2013) Dosimetric Analysis of IMRT versus 3DCRT for Chest Wall Irradiation in Patients with Breast Cancer Using 6MV X-Rays. Indian Journal of Research and Reports in Medical Sciences, 3, 36-39.

[11] Freedman, G.M., Anderson, P.R., Hanlon, A.L., Eisenberg, D., Nicolaou, N. and Li, J. (2004) Intensity Modulated Radiation Therapy (IMRT) Decreases the Acute Skin Toxicity for Large-Breasted Women Receiving Radiation Therapy for Breast Cancer. International Journal of Radiation Oncology, Biology, Physics, 60, S401-S402. https://doi.org/10.1016/j.ijrobp.2004.07.276

[12] Borges, C., Cunha, G., Monteiro-Grillo, I., Vaz, P. and Teixeira, N. (2014) Comparison of Different Breast Planning Techniques and Algorithms for Radiation Therapy Treatment. Physica Medica, 30, 160-170. https://doi.org/10.1016/j.ejmp.2013.04.006

[13] Tanaka, H., Hayashi, S. and Hoshi, H. (2014) Determination of the Optimal Method for the Field-in-Field Technique in Breast Tangential Radiotherapy. Journal of Radiation Research, 55, 769-73. https://doi.org/10.1093/jrr/rrt233 
[14] Sasaoka, M. and Futami, T. (2011) Dosimetric Evaluation of Whole Breast Radiotherapy Using Field-in-Field Technique in Early-Stage Breast Cancer. International Journal of Clinical Oncology, 16, 250-256. https://doi.org/10.1007/s10147-010-0175-1

[15] Emami, B. (2013) Tolerance of Normal Tissue to Therapeutic Radiation. Reports of Radiotherapy and Oncology, 1, 35-48.

[16] Darby, S.C., Ewertz, M., McGale, P., Bennet, A.M., Blom-Goldman, U., Brønnum, D., et al. (2013) Risk of Ischemic Heart Disease in Women after Radiotherapy for Breast Cancer. New England Journal of Medicine, 368, 987-998.

[17] Jin, G.-H., Chen, L.-X., Deng, X.-W., Liu, X.-W., Huang, Y. and Huang, X.-B. (2013) A Comparative Dosimetric Study for Treating Left-Sided Breast Cancer for Small Breast Size Using Five Different Radiotherapy Techniques: Conventional Tangential Field, Filed-in-Filed, Tangential-IMRT, Multi-Beam IMRT and VMAT. Radiation Oncology, 8, Article No. 89. https://doi.org/10.1186/1748-717X-8-89

[18] Zhao, H., He, M., Cheng, G., Han, D., Wu, N., Shi, D., et al. (2015) A Comparative Dosimetric Study of Left Sided Breast Cancer after Breast-Conserving Surgery Treated with VMAT and IMRT. Radiation Oncology, 10, Article No. 231. https://doi.org/10.1186/s13014-015-0531-4

[19] Taylor, C., Correa, C., Duane, F.K., Aznar, M.C., Anderson, S.J., Bergh, J., et al. (2017) Estimating the Risks of Breast Cancer Radiotherapy: Evidence from Modern Radiation Doses to the Lungs and Heart and from Previous Randomized Trials. Journal of Clinical Oncology, 35, 1641-1649. https://doi.org/10.1200/JCO.2016.72.0722

[20] Hall, E.J. (2006) Intensity-Modulated Radiation Therapy, Protons, and the Risk of Second Cancers. International Journal of Radiation Oncology, Biology, Physics, 65, 1-7. https://doi.org/10.1016/j.ijrobp.2006.01.027

[21] Xie, X., Ouyang, S., Wang, H., Yang, W., Jin, H., Hu, B., et al. (2014) Dosimetric Comparison of Left-Sided Whole Breast Irradiation with 3D-CRT, IP-IMRT and Hybrid IMRT. Oncology Reports, 31, 2195-2205.

https://doi.org/10.3892/or.2014.3058 